\title{
Anandamide elevation in cerebrospinal fluid in initial prodromal states of psychosis
}

Dagmar Koethe, * Andrea Giuffrida, * Daniela Schreiber, Martin Hellmich, Frauke Schultze-Lutter, Stefan Ruhrmann, Joachim Klosterkötter, Daniele Piomelli and F. Markus Leweke

\section{Summary}

Anandamide is a bioactive lipid binding to cannabinoid receptors. A homeostatic role for anandamide has been suggested in schizophrenia. We investigated its role in initial prodromal states of psychosis. We measured the levels of anandamide and its structural analog oleoylethanolamide in cerebrospinal fluid and serum of patients in the initial prodromal state $(n=27)$ alongside healthy volunteers $(n=81)$ using high-performance liquid chromatograph/mass spectrometry. Cerebrospinal anandamide levels in patients were significantly elevated. Patients with lower levels showed a higher risk for transiting to psychosis earlier. This anandamidergic up-regulation in the initial prodromal course may suggest a protective role of the endocannabinoid system in early schizophrenia.

\section{Declaration of interest}

\section{None.}

We have previously shown that levels of the endocannabinoid anandamide in cerebrospinal fluid (CSF) in antipsychotic-naive patients with schizophrenia ${ }^{1-3}$ are both markedly elevated and negatively correlated with psychotic symptoms. A model of dopamine/endocannabinoid interaction in acute schizophrenia was proposed where (over-)activation of dopamine $\mathrm{D}_{2}$ receptors is associated with an increased release of anandamide counterbalancing dopamine-mediated psychotic symptoms by strengthening an endogenous feedback loop. This model suggests an adaptation of endocannabinoidergic function over a long period to a slow, gradual increase in dopaminergic neurotransmission. ${ }^{4}$ We investigated whether changes in the endocannabinoid system are already noticeable in initial prodromal states of psychosis and test the hypothesis that an elevation of anandamide in CSF is apparent in this early stage of the disease.

\section{Method}

The ethics committee of the University of Cologne approved the study. All patients and healthy volunteers provided written informed consent.

We consecutively recruited 108 participants, comprising 81 healthy controls with no family history of psychiatric or neurological disorders (56\% male, median age 27.3 years (interquartile range (IQR) 23.9 to 29.9)) and 27 patients with initial prodromal states of psychosis $(70 \%$ male, age 24.3 years (IQR 19.9 to 29.1)). Controls were recruited by word of mouth and were paid for their expenses. Out of the 27 patients, 8 (30\%) received antipsychotic medication.

Controls were screened for current or previous medical and psychiatric disorders. No cannabis use within 6 weeks prior to the study was accepted. Overall, 55 controls (68\%) had taken cannabis $<20$ times/lifetime and $26(32 \%)$ had used cannabis $>20$ but $<50$ times/lifetime.

Patients were assessed at the Cologne Early Recognition Centre using the Positive and Negative Symptom Scale (PANSS), ${ }^{5}$ the Scale of Prodromal Symptoms (SOPS), ${ }^{6}$ and the Schizophrenia Prediction Instrument for Adults (SPI-A). ${ }^{7}$ An initial prodromal state was assumed if any of ten cognitive-perceptive basic symptom or any attenuated or transient psychotic symptom was

*These authors contributed equally to the work. present. After examination, 11 patients (41\%) developed schizophrenia; $36 \%$ had already been treated with antipsychotics before transition. The 75th percentile of time from baseline to transition was 13 months; median follow-up was 61 months (IQR 56 to 80). Overall, 16 patients used cannabis $<20$ times/lifetime and 11 used cannabis $>20$ times/lifetime. All participants showed a negative urine drug screening for illicit drugs.

Cerebrospinal fluid and serum were collected around mid-day using a procedure described elsewhere. ${ }^{2}$ No participants revealed pathognomonic CSF findings according to a standard examination. ${ }^{8}$ We measured CSF and serum levels of anandamide along with oleoylethanolamide, another endogenous fatty acid ethanolamide. These were purified and quantified by isotope dilution high-performance liquid chromatography/mass spectrometry as previously described. ${ }^{9}$

Skewed empirical distributions of continuous variables were summarised by median and IQR (25th to 75th percentile). Independent groups were compared by exact Wilcoxon rank sum test (corrected for ties). Spearman's rho was calculated to assess pairwise correlation of variables. The cumulative probability of transition to psychosis was estimated by the Kaplan-Meier method and compared overall by the log rank test (hazard ratio). Statistical analyses were performed using SPSS and $\mathrm{R}$ for Windows.

\section{Results}

In controls, the median concentration of anandamide in CSF was $<0.001 \mathrm{pmol} / \mathrm{ml}$ (IQR $<0.001$ to 0.005$)$ and $<0.001 \mathrm{pmol} / \mathrm{ml}$ $(<0.001$ to 0.700$)$ in serum. We found no significant correlation between anandamide concentrations in CSF and serum $\left(r_{\mathrm{S}}=0.033\right.$, $P=0.768)$. Median CSF anandamide levels of patients were $0.006 \mathrm{pmol} / \mathrm{ml} \quad(\mathrm{IQR}<0.001$ to $0.073, P=0.004$; online Fig. $\mathrm{DS} 1 \mathrm{a}$ ) and $0.216 \mathrm{pmol} / \mathrm{ml}$ (IQR $<0.001$ to $0.515, P=0.838$ ) in serum. Oleoylethanolamide levels did not differ in CSF or serum between controls (CSF: $0.680 \mathrm{pmol} / \mathrm{ml}$ (IQR 0.280 to 1.100 ); serum: $7.700 \mathrm{pmol} / \mathrm{ml}$ (IQR 4.885 to 11.000$)$ ) and patients (CSF: $0.600 \mathrm{pmol} / \mathrm{ml}$ (IQR 0.315 to 1.026 ), $P=0.896$, online Fig. DS1b; serum: $6.641 \mathrm{pmol} / \mathrm{ml}$ (IQR 2.867 to 9.891), $P=0.139$ ).

A significant inverse correlation between anandamide in CSF and symptoms in patients was observed for PANSS factor 3 
'cognitive syndrome' $\left(r_{\mathrm{S}}=-0.533, P=0.005\right)$. No other psychopathological measures correlated with ethanolamide levels.

In this sample, no relevant differences for CSF and serum levels of anandamide and oleoylethonalamide were found between patients and controls regarding smoking, cannabis use, medication or body mass index. No significant difference was found for all ethanolamide levels between medicated and antipsychotic-naive patients. Interestingly, patients with cannabis use $<20$ times/lifetime showed no significantly different ethanolamide levels compared with those with $>20$ times/lifetime use.

By splitting patients at the median CSF anandamide value $(0.006 \mathrm{pmol} / \mathrm{ml})$ into low and high groups, patients with higher anandamide levels in CSF were found to be associated with an extended transition time into frank psychosis $(P=0.095$; online Fig. DS1c). The relative risk of transition of individuals in the high $v$. low anandamide groups was 0.33 (95\% CI 0.09-1.29). A similar analysis for lifetime cannabis use was not associated with the likelihood of transition to psychosis.

\section{Discussion}

This is the first study investigating levels of anandamide and oleoylethanolamide in CSF and serum of patients with initial prodromal states of psychosis. We found levels of anandamide in CSF significantly elevated in patients. The anandamide elevation was comparable to that described for first-onset, antipsychotic-naive schizophrenia ${ }^{2}$ and was found in CSF exclusively, making its central origin most likely. As oleoylethanolamide concentrations did not differ significantly between patients and controls, it is unlikely that changes in anandamide levels were caused by a generalised alteration in ethanolamide signalling.

Interestingly, we found a trend for an association of anandamide elevation in CSF with a longer time to psychosis transition in patients; this trend, however, did not reach statistical significance mainly owing to lack of power. This is in line with our hypothesis of an adaptive role for anandamide in schizophrenia, indicating that those patients expressing higher CSF anandamide are less likely to have psychotic symptoms. Four patients who transited to schizophrenia were treated with dopamine $\mathrm{D}_{2-}$ antagonists at examination and showed no significantly different ethanolamide levels compared with antipsychotic-naive patients. However, the observation that some patients did not show significant changes in CSF anandamide levels might imply a fluctuation or state-dependency of the alterations of the endocannabinoid system during the initial prodromal period. This has been recently suggested for other markers in CSF. ${ }^{10}$ Alternatively, this may be related to the particular condition investigated, as not all individuals diagnosed with an initial prodromal state develop schizophrenia but may either return to a normal psychiatric status or develop another psychiatric disorder. ${ }^{11}$ It is interesting to note that in this sample a history of cannabis use did not have an impact on levels of CSF anandamide as previously reported in acute antipsychotic-naive schizophrenia. ${ }^{12}$ Further, no relation between history of cannabis use and risk of transition to psychosis was observed. Both aspects may be related to the relationship between $\Delta^{9}$-tetrahydrocannabinol and cannabidiol in street cannabis consumed, which was not further evaluated. ${ }^{13}$

Our findings suggest that endogenous anandamide mobilisation may play a protective role in at least a subgroup of patients with early stage schizophrenia. ${ }^{4}$ Based on this observation, it may be hypothesised that assaying the endocannabinoids in CSF may provide useful biomarkers in early schizophrenia.
Dagmar Koethe, MD, Department of Psychiatry and Psychotherapy, University of Cologne, Germany; Andrea Giuffrida, PhD, Department of Pharmacology, University of Texas Health Science Center, San Antonio, Texas, USA:

Daniela Schreiber, PhD, Department of Psychiatry and Psychotherapy, University of Cologne, Germany, and Departments of Pharmacology and Biological Chemistry, University of California, Irvine, California, USA; Martin Hellmich, MDSci, Institute of Medical Statistics, Informatics and Epidemiology, University of Cologne, Germany; Frauke Schultze-Lutter, PhD, Stefan Ruhrmann, MD, Joachim Klosterkötter MD, Department of Psychiatry and Psychotherapy, University of Cologne, Germany; Daniele Piomelli, DPharm, PhD, Departments of Pharmacology and Biological Chemistry, University of California, Irvine, California, USA; F. Markus Leweke, MD, Department of Psychiatry and Psychotherapy, University of Cologne, and Central Institute of Mental Health, Mannheim, University of Heidelberg, Germany

Correspondence: Dr. F. Markus Leweke, Department of Psychiatry and Psychotherapy, Central Institute of Mental Health, J5, 68159 Mannheim, Germany. Email: leweke@cimh.de

First received 16 Apr 2008, final revision 30 Jun 2008, accepted 1 Sep 2008

\section{Funding}

The study was funded by the Stanley Medical Research Institute (01-315 and 03-NV-003 to F.M.L.), the Koeln Fortune Program (108-2000 to F.M.L.) and the Federal Ministry of Education and Research (BMBF) (01KN0706 to D.K.)

\section{Acknowledgements}

We thank Drs Brit M. Nolden, Christoph W. Gerth, Christian Mauss and Johannes Faulhaber for collection of data.

\section{References}

1 Leweke FM, Giuffrida A, Wurster U, Emrich HM, Piomelli D. Elevated endogenous cannabinoids in schizophrenia. Neuroreport 1999; 10: 1665-9.

2 Giuffrida A, Leweke FM, Gerth CW, Schreiber D, Koethe D, Faulhaber J, et al. Cerebrospinal anandamide levels are elevated in acute schizophrenia and are inversely correlated with psychotic symptoms. Neuropsychopharmacology 2004; 29: 2108-14.

3 Leweke FM, Giuffrida A, Koethe D, Schreiber D, Nolden BM, Neatby MA, et al. Anandamide levels in cerebrospinal fluid of first-episode schizophrenic patients: impact of cannabis use. Schizophr Res 2007; 94: 29-36.

4 Leweke FM, Koethe D. Cannabis and psychiatric disorders: it is not only addiction. Addict Biol 2008; 13: 264-75.

5 Kay SR, Fiszbein A, Opler LA. The positive and negative syndrome scale (PANSS) for schizophrenia. Schizophr Bull 1987; 13: 261-76.

6 Ruhrmann S, Klosterkötter J. Schizophrenia Proneness Instrument. Adult Version (SPI-A). Giovanni Pioriti s.r.I., 2007.

7 Miller TJ, McGlashan TH, Woods SW, Stein K, Driesen N, Corcoran CM, et al. Symptom assessment in schizophrenic prodromal states. Psychiatric Q 1999; 70: $273-87$.

8 Andersson $\mathrm{M}$, Alvarez-Cermeno J, Bernardi G, Cogato I, Fredman $\mathrm{P}$, Frederiksen J, et al. Cerebrospinal fluid in the diagnosis of multiple sclerosis: a consensus report. J Neurol Neurosurg Psychiatry 1994; 57: 897-902.

9 Schreiber D, Harlfinger S, Nolden BM, Gerth CW, Schömig E, Klosterkötter J, et al. Determination of anandamide and other fatty acylethanolamides in human serum by electrospray tandem mass spectrometry. Anal Biochem 2007; 361: $162-8$

10 Huang JT, Leweke FM, Tsang TM, Koethe D, Kranaster L, Gerth CW, et al. CSF metabolic and proteomic profiles in patients prodromal for psychosis. PLOS ONE 2007; 2: e756.

11 Corcoran C, Malaspina D, Hercher L. Prodromal interventions for schizophrenia vulnerability: the risks of being 'at risk'. Schizophr Res 2005; 73: 173-84.

12 Leweke FM, Giuffrida A, Koethe D, Schreiber D, Nolden BM, Kranaster L, et al. Anandamide levels in cerebrospinal fluid of first-episode schizophrenic patients: impact of cannabis use. Schizophr Res 2007; 94: 29-36.

13 Morgan CJA, Curran HV. Effects of cannabidiol on schizophrenia-like symptoms in people who use cannabis. Br J Psychiatry 2008; 192: 306-7. 